\title{
Design of Embedded Industrial robot based on PLC servo control
}

\author{
JieYuhan \\ The Two Branch School of Ethnic Education,Nanchang Institute of Science \&Technology,Nanchang \\ 330108, China
}

\begin{abstract}
Keywords: industrial robot; automatic positioning; servo motor; PLC programming; Modbus communication
\end{abstract}

\begin{abstract}
In order to improve the automation degree of robot, design size of the robot is reduced, a new industrial robot has been designed using PLC, servo motor drive and servo motor as the core component, through the PLC programming and the Modbus communication. IPC and Delta PLC of this kind of robot uses RS232 serial port and communication mode of ASCII protocol to control servo motor, force control software and servo driver use Modbus protocol communication mode for real-time monitoring of motor movement state. Through the PLC programming, the positioning function of the industrial robot servo motor is realized. Finally, the system positioning accuracy is higher after the debugging and running, which can meet the requirements for embedded industrial robot design.
\end{abstract}

\section{Introduction}

Servo motor is one of the most commonly used control devices in the industrial robot automation control system. Because the servo motor has fast response and high control precision, which is used in many automatic control occasions. It will promote the level of automatic control and remote monitoring system that combining the servo motor and bus communication control technology. Now the industrial automation control has changed from the single chip microcomputer control to the integrated control system. With the arrival of network times, it provides a great convenience to the industrial automation control network. Modbus communication protocol is one of the protocols of industrial control network. Through this protocol, the network can be used to connect the controller, the communication between the systems is realized. It will effectively improve the control efficiency and accuracy of the robot if Modbus communication and PLC program has embedded in the industrial robot control system.

\section{PLC communication control of embedded industrial robot}

Communication is the foundation of realizing the automation control of embedded industrial robot, the embedded industrial robot based on PLC programming is communicated mainly by two ports $\mathrm{CN} 2$ and $\mathrm{CN} 3$, the communication principle of $\mathrm{CN} 2$ port is shown in Fig.1. 


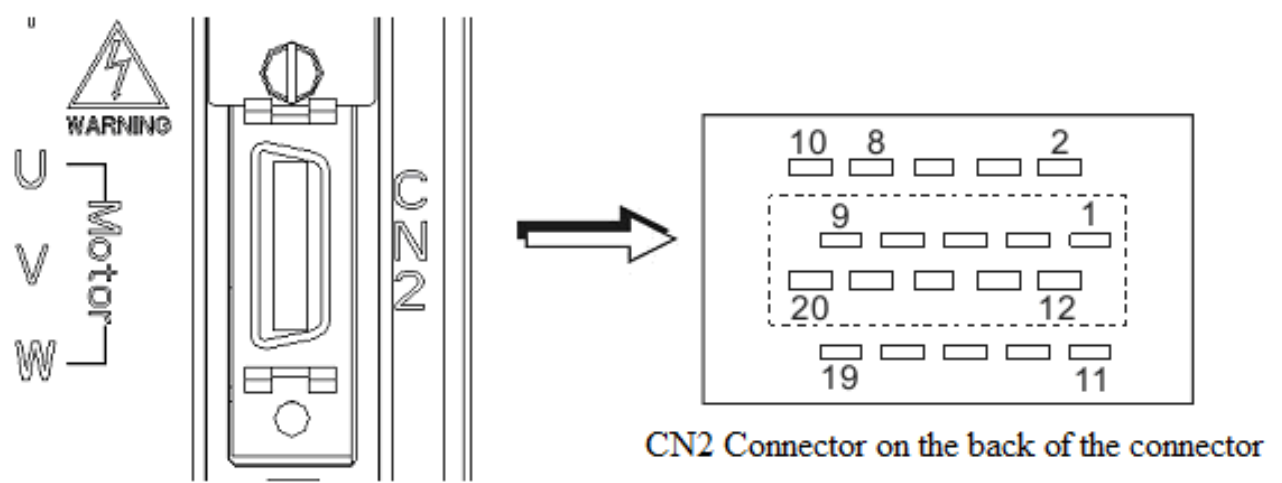

Fig.1 Schematic diagram of CN2 port

Servo motor driver CN2 is the encoder signal interface of motor speed, ECMA servo motor driver series has a built-in encoder, its form as shown in Fig.1, which is 2500pprA, B, Z, U, V, W. When the power is started, the signal in the way of six kinds of $\mathrm{U}+, \mathrm{V}+, \mathrm{W}+, \mathrm{U}-, \mathrm{V}-, \mathrm{W}-$ to inform the servo motor drive in 0.5 seconds, and then six lines are replaced by A+, B+, Z+, A-, B-, Zsignals. The encoder signal interface has a total of 10 lines plus the two power line and two wires.

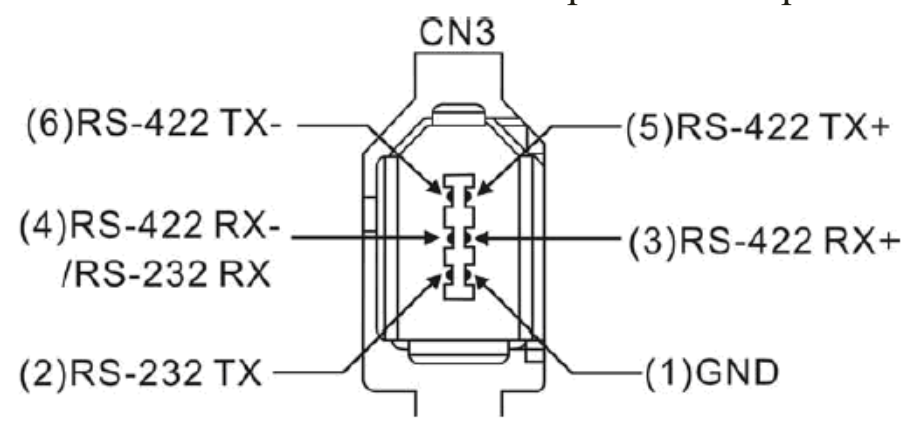

Fig.2 The CN3 port

$\mathrm{CN} 3$ is the communication interface of the servo motor driver, which is used to connect the servo motor and the computer by the driver, and the special software is used to communicate with the computer by MODBUS protocol. This type of driver provides three communication modes, including RS-232, RS-485, RS-42, which can use (P3-05) to set. The RS-232 serial communication is used in the paper, which can feedback the motor speed and other motion state in the form of signal, its wiring is shown in Fig.3.

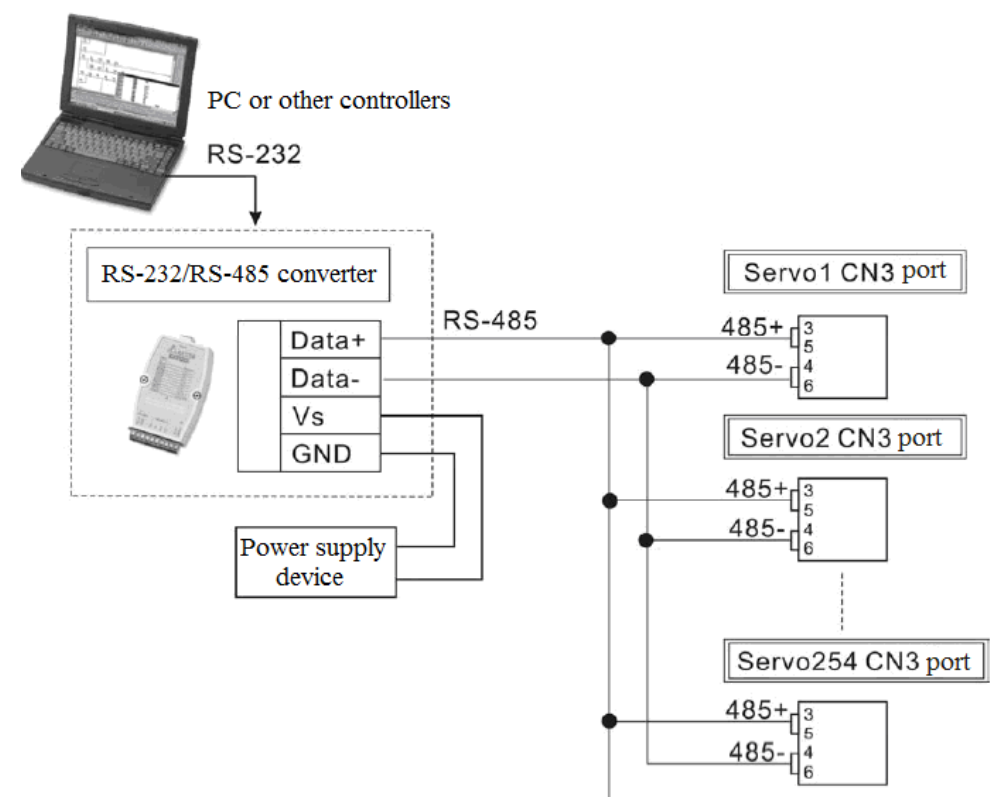

Fig.3 Schematic diagram of Modbus communication 
Fig. 3 shows the Modbus communication connection diagram, the industrial robot based on PLC and servo control takes the RS232 serial port and ASCII protocols for communication, RS232 port connect industrial robot and $\mathrm{PC}$, the basic communication function is realized by connection of RS-485 port and the lower position machine.

\section{Positioning program design of industrial robot based on PLC}

The positioning process is the most commonly used process by Industrial robot, the positioning fuction is divided into absolute positioning and relative positioning, the method of relative positioning is mainly used in the preparation of the PLC industrial robot positioning program, which can make the robot automatically positioning function.

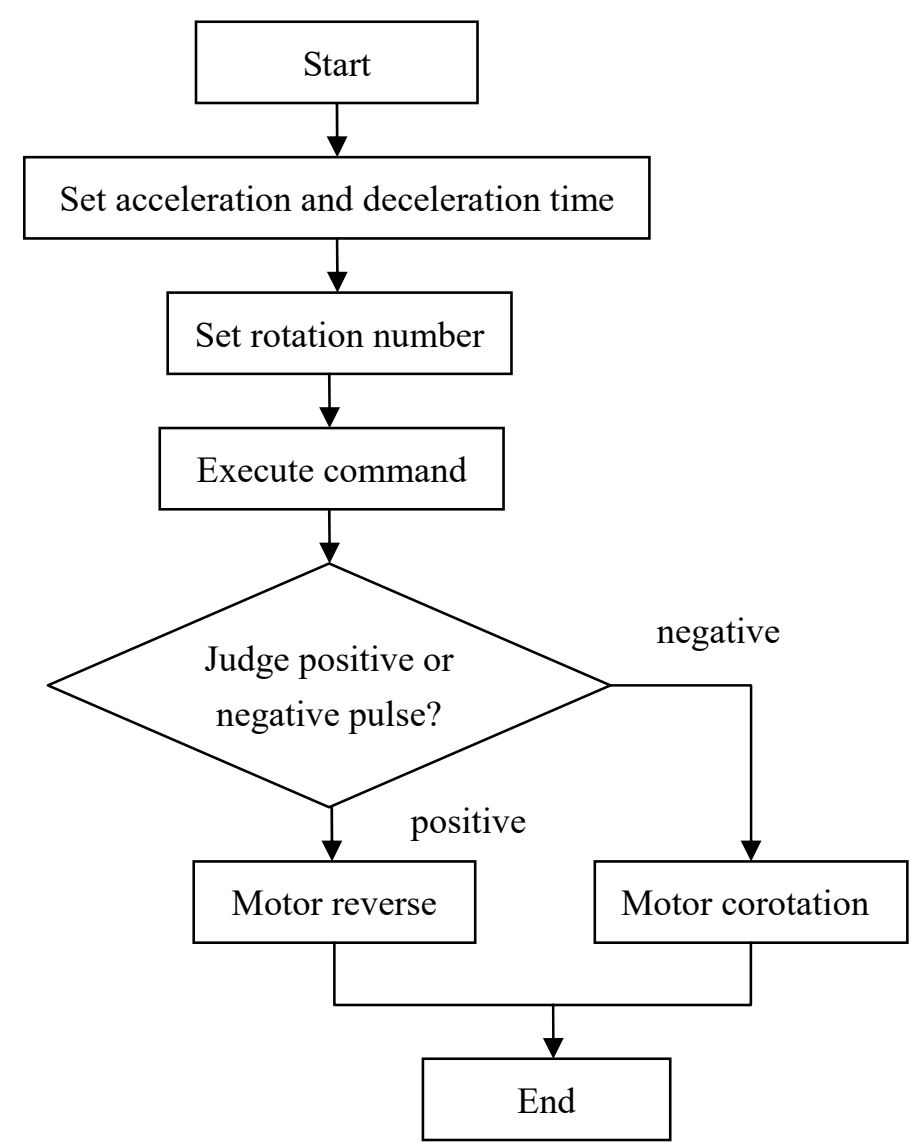

Fig. 4 The design process of positioning program

Figure 4 shows the specific design process of positioning program, the main process is to startup program, and then set acceleration and deceleration time, and set the number of rotations and start the execution of commands, and then pulse judgment, so as to realize the motion state control of the motor, the PLC program is shown in Fig.5.

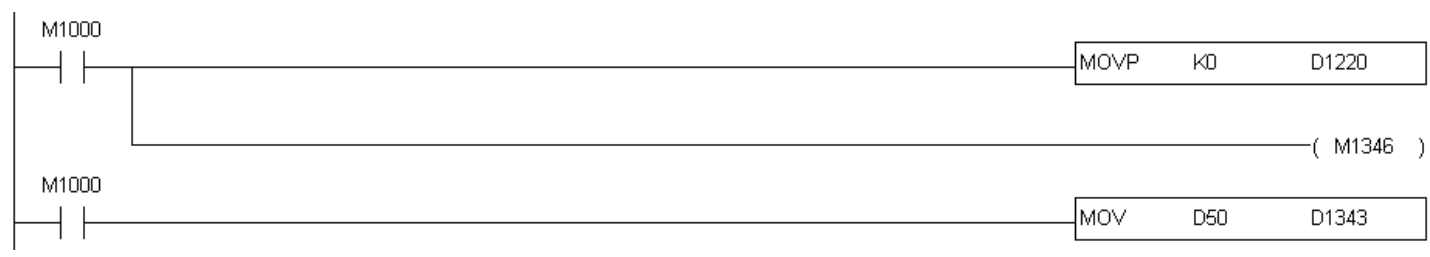

Fig. 5 PLC positioning program

In order to realize the motor relative positioning function, using the D13 storage to save the corotation circle number, and convert the number of cycles to pulses stored in D19, then save the corotation speed in the storage D26, and then convert the speed to pulse signal, which can store in 
the D66 register, so as to realize the positioning function of industrial robots, the PLC program is shown in Figure 6.

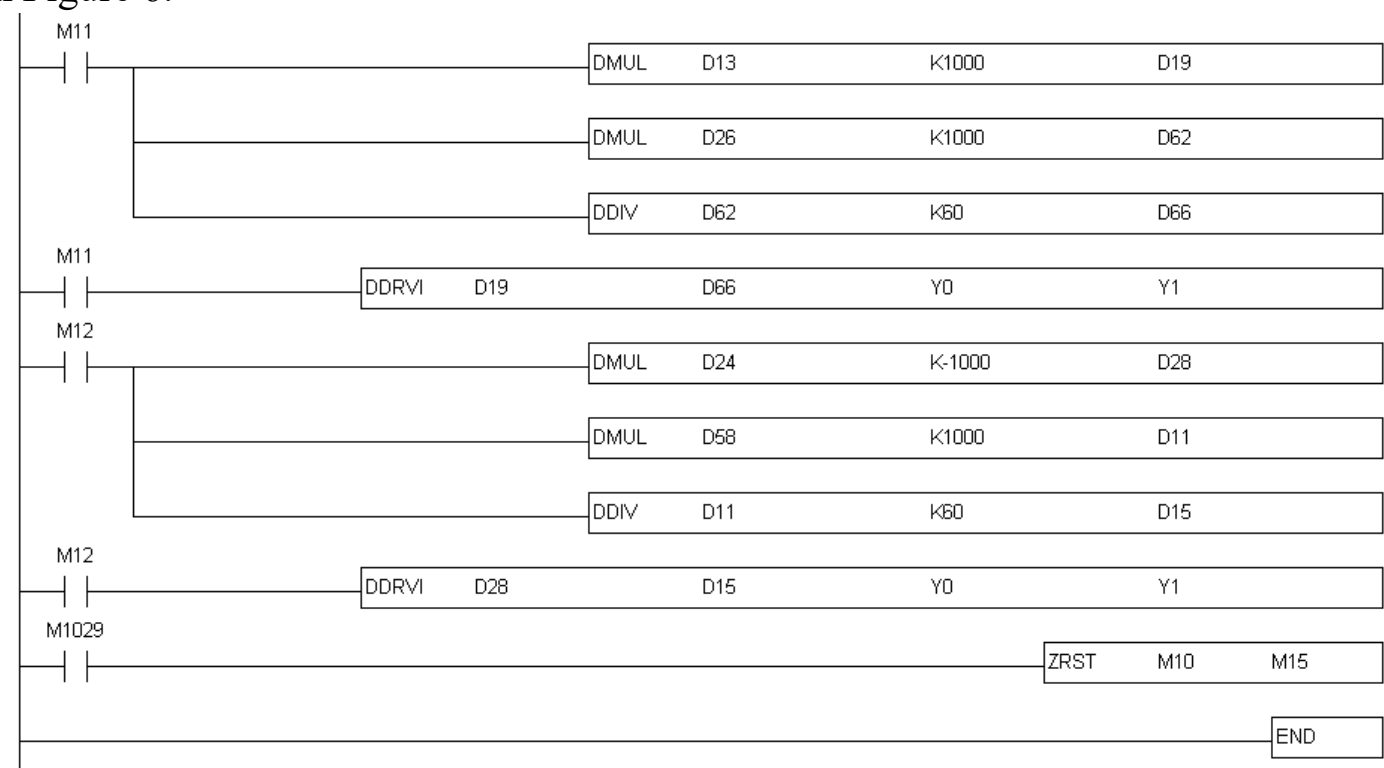

Fig. 6 The relative positioning program

In order to verify the validity of program, the performance of the servo motor was tested, Fig. 7 is encoder feedback curve in the location process of the running motor, motor speed increases from 0 to $100 \mathrm{r} / \mathrm{min}$ then has the constant speed. When the DAC voltage is less than a certain value, motor gradually goes into the positioning process, , the motor rotational speed becomes 0 after the end of positioning.

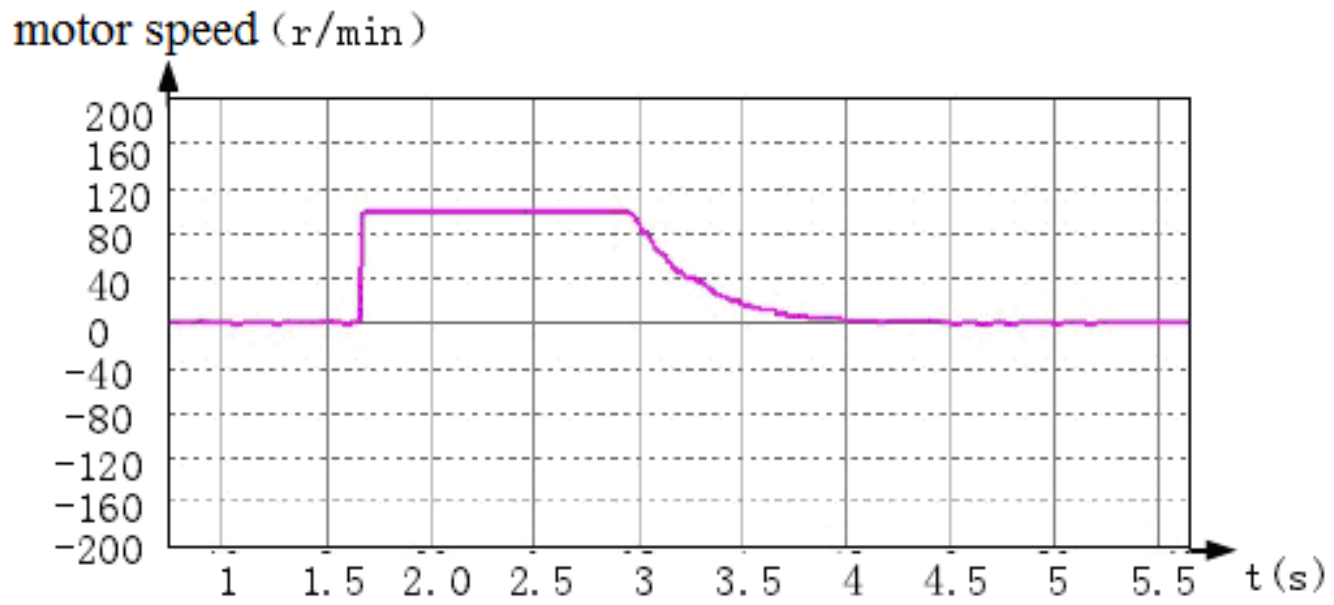

Fig. 7 The speed chart of motor positioning process

We set the ratio of input and output of embedded industrial robot servo system to 1:10. When the input is 500, and the output is5000 encoder pulse, we statistics the coded pulse value of the ideal output and the actual output in order to test the accuracy of system, which as shown in Table 1.

Tab.1 Statistic results of input and output

\begin{tabular}{|c|c|c|c|}
\hline Test code & input & ideal output & actual output \\
\hline 1 & 500 & 5000 & 5001 \\
\hline 2 & 600 & 6000 & 6002 \\
\hline 3 & 700 & 7000 & 7001 \\
\hline 4 & 800 & 8000 & 8002 \\
\hline 5 & 900 & 9000 & 9003 \\
\hline
\end{tabular}


Table 1 shows the coding statistical results of actual motor input and ideal and actual output, it can be seen by test results in Ttab.1, that anastomosis degree of actual output and the desired output is high, and the maximum error value of the ideal output only three coding, which verifies the validity and reliability of PLC program.

\section{Conclusion}

A new embedded industrial robot has been designed in this paper, based on the control principle of the embedded system, using the PLC programming and the Modbus communication technology. RS232 serial port and MODBUS ASCII communication protocol have been used in the robot communication, the positioning function of the robot is realized by PLC programming approach. Speed curve of motor positioning process and the coding statistical results of actual motor input and ideal and actual output have been obtained by test of servo control system, the test results show that the maximum value of the error between the ideal output is only three pulse code, high positioning accuracy to meet needs of industrial robot design.

\section{Acknowledgement}

The work was supported by the the key subject of science and technology project of Jiangxi Education Department with the project number GJJ151235 and the project name Research and Realization of Electric Control System in Alcohol Hydrogen Power System Device Based on PLC.

\section{Reference}

[1] Wang Tianmiao, Tao Yong. Industrial robot technology status and industrialization development strategy in China [J]. Journal of Mechanical Engineering, 2014,50 (9): 1-12. Tension

[2] Li Ruifeng. Industrial robot industry development strategy in China [J]. Aeronautical Manufacturing Technology, 2010, (9): 32-37.

[3] Wan Lin, Tang Houjun, He Chao, Huang Weifang. Design and development of embedded open motion controller based on ARM and FPGA [J]. Electrical Automation, 2011,33 (3): 59:61.

[4] Si Yuxian. Introduce to leading manufacturers of intelligent robot: Fanuc[J]. DIANZI YU DIANNAO, 2011, (12): 48-49.

[5] Luo Weitao, Zhang Tie. Research on robot motion controller based on ARM+DSP+FPGA [J]. Machine tool \& Hydraulic, 2011,39 (15): 55-58.

[6] Huang Zhen, Guo Rifeng. Design of asynchronous serial bus based on FPGA [J]. Electronic Component \& Device Applications, 2012,14 (2): 26-29.

[7] Lin Yongqiang, Wang Yong, Feng Yizhao. Open motion controller technology status and development trend [J]. Information Technology, 2010 (005):184-186.

[8] Wang Weimin, Li Yinhua, Zhang Jin. Research on the open servo motion controller based on DSP+FPGA [J]. Industrial Control Computer, 2006, 19 (1):58-59.

[9] Ceng Xiangyu, Sun Hanxu, Jia Qingxuan. Design and application of embedded multi core host controller for modular robot [J]. Microcontrollers \& Embedded system, 2012, 12 (5):20-23. 